\title{
THE TRANSVERSE NONLINEAR TUNE SHIFT AS STABILIZING FACTOR IN HALO CREATION IN SPACE CHARGE DOMINATED BEAM*
}

\author{
N.Vasyukhin, Yu.Senichev and R.Tölle, Forschungszentrum Jülich, Germany.
}

\section{Abstract}

One of the most important problems for space charge dominated beams in the low energy part of superconducting linacs is halo creation. Many authors show one of the key effects in halo creatiation is parametric resonance due to the mismatched betafunction oscillation (between core and particle). To estimate parametric resonance conditions the nonlinear tune shift for binomial distributed beam is described theoretically in this article. Simultaneously the beam dynamics simulation 3D PIC code was developed. The transverse oscillation frequencies are compared with parametric resonance criteria. As a result the recommendation for space charge shift is concluded to minimize halo creation.

\section{EQUATION OF MOTION}

There are two different concepts explaining the halo creation: mechanical and thermodynamical. In linear accelerators the acceleration time is much shorter than the relaxation time of the system. Therefore a mechanical concept is more appropriate than a thermodynamical one. The development of this concept was attempted by $\mathrm{Yu}$. Senichev [1]. The point was to describe qualitatively nonlinear parametric resonances due to mismatched RMS size of bunch and forced envelope oscillations in alternate gradient focusing structure. Obviously, this estimation can be done only for the "frozen core" of bunch or for the case, when the redistribution in halo does not affect on the core, and the resonance conditions cause increasing the transverse oscillations of particles in halo only. We used the isolated single resonance approach.

To solve the equation analytically the following approximations are taken: axial symmetry, binomial distribution of particles, smooth approximation and "frozen" space charge forces. In order to clarify the overall picture of halo formation mechanism shortened derivations of equations are carried out. Firstly one can write the general equation of radial motion for arbitrary particle considering axial symmetry of RF field:

$$
\begin{aligned}
\frac{d^{2} r}{d z^{2}}=-\frac{e \cdot k_{1} \cdot E_{1}}{4 m_{0} c^{2} \gamma^{3} \beta_{q s}^{2}} \cdot \sin \varphi_{q s} \cdot r & +\frac{e c G(z)}{m_{0} c^{2} \beta_{q s} \gamma} x+ \\
& +\frac{F_{s c}}{m_{0} c^{2} \beta_{q s}^{2} \gamma}
\end{aligned}
$$

where $k_{1}=\frac{2 \pi}{\beta_{s r} \lambda}, \mathrm{E} 1-$ accelerating harmonic amplitude, $\mathrm{G}(\mathrm{z})$ - function describing quadrupoles gradient, Fsc space charge force, $\varphi$ qs - particle phase, $\beta \mathrm{qs}-$ particle

*Work supported by the European Community-Research Infrastructure Activity under the FP6 "Structuring the European Research Area" program (CARE, contract number RII3-CT-2003-506395) velocity, $\beta$ str -accelerating structure phase velocity.

In case of binomial distribution the particle density can be written as:

$$
\rho_{s c}(r)=\frac{e n m}{\pi r_{0}^{2} l}\left[1-\frac{r^{2}}{r_{0}^{2}}\right]^{m-1}
$$

where $\mathrm{m}$ - order of binomial distribution, $r_{0}$ - envelope of the beam which is connected with RMS deviation by the ratio $\sigma^{2}=\frac{r_{0}^{2}}{2 m+2}$. From this it follows that space charge force acting at arbitrary particle $F=e(E-v B)$ can be found from Poisson equation:

$$
F_{s c}(r)=\frac{e^{2} n}{2 \pi \varepsilon_{0} \gamma^{2} l r}\left\{1-\left[1-\frac{r^{2}}{r_{0}^{2}}\right]^{m}\right\}
$$

where $\mathrm{n}$ - number of particles in the bunch, 1 - length of the bunch. Due to the fact that the equation of motion includes quadrupole focusing term it is convenient to pass to new longitudinal coordinates $s=z / S$ normalized to focusing period S. Substituting (2) in (1) and designating $C_{s c}=\frac{e^{2} n S^{2}}{2 \pi \varepsilon_{0} m_{0} c^{2} \beta_{q}^{2} \gamma^{3} l}$ and $Q(z)=\frac{e \cdot S^{2} \cdot k_{1} \cdot E_{1}}{4 m_{0} c^{2} \gamma^{3} \beta_{q s}^{2}} \cdot \sin \varphi_{\varphi r}-\frac{e \cdot S^{2} \cdot G(z)}{m_{0} c \beta_{q u} \gamma}$ one can derive from (1):

$$
\ddot{r}+Q(s) r=\frac{C_{s c}}{r}\left(1-\left(1-\frac{r^{2}}{r_{0}^{2}}\right)^{m}\right)=C_{s c} \sum_{n=1}^{m} \frac{(-1)^{n-1} m !}{(m-n) ! n ! r_{0}^{2 n}} r^{2 n-1}
$$

As soon as $\mathrm{Q}(\mathrm{s})$ is periodical function the solution should be periodical too. Following Courant-Snyder formalism one can represent the solution as:

$$
r(s)=A \beta^{1 / 2}(s) \eta(s)
$$

where A - initial amplitude of oscillation, for envelope $A=\sqrt{\varepsilon}, \beta-$ beta function, $\eta-$ oscillating function. It is important to note that if the motion equation in dimensionless coordinates $s$ then beta function should be also dimensionless and emittance dimension is $\mathrm{m}^{2}$. The envelope can be represented as $r_{0}(s)=\varepsilon^{1 / 2} \beta^{1 / 2}(s)$. Substituting this and (3) to the equation of motion and designating $\quad c_{n}=\frac{(-1)^{n-1} m ! A^{2 n-2}}{(m-n) ! n ! \varepsilon^{n}} \quad$ one can derive:

$$
\begin{array}{r}
\beta^{1 / 2} \ddot{\eta}+\frac{\dot{\beta} \dot{\eta}}{\beta^{1 / 2}}+\left(\frac{d^{2}\left(\beta^{1 / 2}\right)}{d s^{2}}+Q(s) \beta^{1 / 2}-\frac{C_{s c} m}{\beta^{1 / 2} \varepsilon}\right) \eta= \\
=\frac{C_{s c}}{\beta^{1 / 2}} \sum_{n=2}^{m} c_{n} \eta^{2 n-1}
\end{array}
$$

Here the linear part of the space charge force is transported to the left-hand side of equation. In order to solve this inhomogeneous differential equation firstly it is necessary to solve the homogeneous equation. 


\section{Homogeneous Motion Equation Solution}

The solution for $\eta$ of homogeneous equation will be considered in the form $a \cdot e^{i \varphi}$. Substituting this in homogeneous equation of motion and separating imaginary and real part one can obtain the equation system:

$$
\left\{\begin{array}{l}
\eta=e^{i j \frac{d z}{\beta}} \\
\frac{d^{2}\left(\beta^{1 / 2}\right)}{d s^{2}}+Q(s) \beta^{1 / 2}-\frac{C_{s c} m}{\beta^{1 / 2} \varepsilon}=\frac{1}{\beta^{3 / 2}}
\end{array}\right.
$$

The first equation reflects the Floquet phase dependency on Floquet amplitude and the second equation is the well known envelope equation. Now let's solve the envelope equation in smooth approximation

$$
A \beta^{1 / 2}(s)=R(s)(1+q(s))
$$

where $R(s)$ - slow function, $q(s)$ - fast oscillating function. After averaging by focusing period one can obtain averaged envelope equation in smooth approximation:

$$
\ddot{R}+\mu_{0}^{2} R-\frac{A^{4}}{R^{3}}-\frac{A^{2} C_{s c} m}{R \varepsilon}=0
$$

The stationary solution will be at $\ddot{R}=0$ :

$$
R_{c}=\frac{A}{\sqrt{\mu_{0}}} \sqrt{h+\sqrt{h^{2}+1}}
$$

where $h=\frac{C_{s c} m}{2 \varepsilon \mu_{0}}-$ analogue of Kapchinskiy's parameter. Now let's consider behaviour of mismatched beam using perturbation theory $R(s)=R_{c}+\Delta r(s)$. Substituting this to (6) one can find that the solution for perturbation can be represented as:

$$
\Delta r=r_{m} \cos \left(\mu_{0} \sqrt{2+\frac{2}{\left(h+\sqrt{h^{2}+1}\right)^{2}}} s\right)=r_{m} \cos (\tilde{\mu} s)
$$

where $r_{m}$ - amplitude of mismatching, $\tilde{\mu}$ - frequency of perturbed envelope oscillation. In case of extremely high currents the frequency of envelope oscillations is approaches to $\mu_{0} \sqrt{2}$, while the frequency of particle oscillations in the centre of bunch is approaches to null. Substituting (7) and (6) into (5) one can write the approximate solution for beta function:

$$
A^{2} \beta \approx R_{c}^{2}+2 R_{c} r_{m} \cos (\tilde{\mu} s)+2 R_{c}^{2} q
$$

Thus, the approximate solution of homogeneous motion equation considering linear space charge force is found.

\section{Inhomogeneous Motion Equation Solution}

Unfortunately, the only clear way to solve the inhomogeneous motion equation is to abandon the Floquet functions. Considering that the perturbation is small it is possible to substitute second equation of (4) to inhomogeneous equation. In order to exclude first derivation term the equation can be written in new coordinate $\tau=\int_{0}^{s} \frac{d s}{\beta}$ :

$$
\frac{d^{2} \eta}{d \tau^{2}}+\eta=\beta C_{s c} \sum_{n=2}^{m} c_{n} \eta^{2 n-1}
$$

The complexity lies in that the derivatives in this equation are taken by $\tau$ but right-hand side is depending on $s$. Considering periodical solution $r(s)=r(s+1)$ let's represent $\tau$ in averaged form $\tau(s)=\mu \cdot s$, where $\mu=\int_{s}^{s+1} \frac{d \zeta}{\beta(\zeta)}-$ average oscillation frequency. In this case the motion equation system can be rewritten:

$$
\left\{\begin{array}{l}
\frac{d^{2}\left(\beta^{1 / 2}\right)}{d s^{2}}+Q(s) \beta^{1 / 2}-\frac{C_{s c} m}{\beta^{1 / 2} \varepsilon}=\frac{1}{\beta^{3 / 2}} \\
\ddot{\eta}+\mu^{2} \eta=\mu^{2} \beta C_{s c} \sum_{n=2}^{m} c_{n} \eta^{2 n-1}
\end{array}\right.
$$

Now let's substitute (8) in the second equation of this motion equation system:

$$
\ddot{\eta}+\mu^{2} \eta=\mu^{2}\left(\frac{R_{c}^{2}}{A^{2}}+\frac{2 R_{c} r_{m}}{A^{2}} \cos (\widetilde{\mu} s)+\frac{2 R_{c}^{2}}{A^{2}} q\right) \frac{C_{s c}}{A^{2}} \sum_{n=2}^{m} c_{n} \eta^{2 n-1}
$$

The solution for this equation can be found using asymptotical methods of Bogolubov-Mitropol'skij [2]. Following this asymptotical method $\eta$ can be represented as $\eta=a \cos (\theta)$, where in $a$ and $\theta$ are in first approximation defined as:

$$
\left\{\begin{array}{c}
\frac{d a}{d s}=-\mu M\left[\left(R_{c}^{2}+2 R_{c} r_{m} \cos (\tilde{\mu} s)+2 R_{c}^{2} q\right) .\right. \\
\left.\cdot \frac{C_{s c}}{A^{2}} \sum_{n=2}^{m} c_{n} a^{2 n-1} \cos ^{2 n-1}(\theta) \sin (\theta)\right] \\
\frac{d \theta}{d s}=\mu-\frac{\mu}{a} M\left[\left(R_{c}^{2}+2 R_{c} r_{m} \cos (\tilde{\mu} s)+2 R_{c}^{2} q\right) .\right. \\
\left.\cdot \frac{C_{s c}}{A^{2}} \sum_{n=2}^{m} c_{n} a^{2 n-1} \cos ^{2 n-1}(\theta) \cos (\theta)\right]
\end{array}\right.
$$

Here $M[f(\theta)]=\frac{1}{T} \int_{0}^{T} f(\theta) d \theta-$ is the averaging operator. The first term in the right-hand side of the first equation is proportional to $R_{c}^{2}$ and after averaging it is equal zero because of orthogonal property for $\cos ^{2 n-1}(\theta)$ and $\sin (\theta)$. The second term in the right-hand side of the second equation contains constant constituent in expansion of $\cos ^{2 n}(\theta)$ and this constant can be taken out from averaging operator. Thus, two oscillating components remain under averaging operator in both equations.

\section{Resonances Caused by Beta Function Modulation}

Examining (9) one can note that in order to perform averaging [2] it is necessary to introduce a slow phase. As soon as oscillating components under averaging operator 
have different frequencies, the choice of considered resonance type should be done. First the resonance caused by mismatched beta function oscillation will be considered. Therefore $\mathrm{q}(\mathrm{s})$ function can be omitted expecting that frequencies of harmonics from $\mathrm{q}(\mathrm{s})$ function expansion are quite far from frequencies of nonlinear components binomial expansion and it does not contribute to the averaged value of right-hand side (9). The slow phase $\psi$ can be introduced as:

$$
2 n_{r} \psi=\left(2 n_{r}-2 k\right) \theta-\tilde{\mu} s
$$

Now, considering isolated resonance condition $\tilde{\mu} \approx\left(2 n_{r}-2 k\right) \dot{\theta}_{s}$, passing to the coordinates motion-angle $a^{2}=I$ and remaining the biggest item in binomial expansion $\mathrm{k}=0$ one can write updated equation system. Supposing that it is canonical equation system the Hamiltonian can be written:

$$
\begin{aligned}
H=\left(\mu-\frac{\tilde{\mu}}{2 n_{r}}\right) I- & R_{c}^{2} C_{s c} A^{-2} \sum_{n=2}^{m} \frac{1}{n} c_{n} I^{n} G_{2 n}- \\
& -\frac{\mu}{n_{r}} R_{c} r_{m} C_{s c} A^{-2} c_{n_{r}} I^{n_{r}} d_{n_{r} 0} \cos \left(2 n_{r} \psi\right)
\end{aligned}
$$

This Hamiltonian describes the averaged transverse motion in vicinity of the $n_{r}$ nonlinear resonance for an arbitrary particle. Taking Tailor in a resonance point $I=I_{0}$ one can find for the Hamiltonian perturbation $H_{r}(\delta I, \psi)=H(\delta I, \psi)-H_{0}\left(I_{0}\right):$

$$
\begin{aligned}
H_{r}=-\frac{\mu R_{c}^{2} C_{s c}}{2 A^{2}} & R_{c} \sum_{k=2}^{m}(k-1) c_{k} I_{0}^{k-2} G_{2 k}(\delta I)^{2}- \\
& -\frac{R_{c} C_{s c} r_{m}}{n_{r} A^{2}} c_{n_{r}} I_{0}^{n_{r}} d_{n_{r} 0} \cos \left(2 n_{r} \psi\right)
\end{aligned}
$$

and for the separatrix size when particles will be captured in resonance:

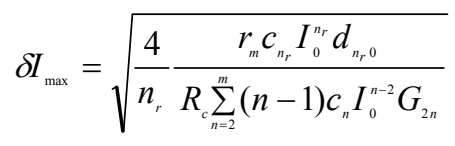

Amplitude of transverse oscillation increases for captured particles, but with increasing of amplitude the nonlinear tune shift leads the particles away from the resonance.

Thus, the mechanical approach describes the system in the non-linear space charge resonance with the "frozen core". However, the particles involved in the resonance are redistributed, and they try to destroy the resonance, taking the more smooth distribution. Nevertheless, this approach describes the halo growth in initial stage transforming into the stationary distribution, and it is the good quantitative and qualitative evaluation of halo growth stabilized on the fixed level.

\section{COMPUTATIONAL RESULTS}

Using modern calculation platforms it is possible to substitute practical values in order to observe Hamiltonian transformation. For instance, the above described Hamiltonian was calculated in MathCAD ${ }^{\circledR}$ for the
COSY-Injector accelerating channel [3] with $17 \mathrm{~mA}$ average current and $10 \%$ mismatch (see Fig. 1).

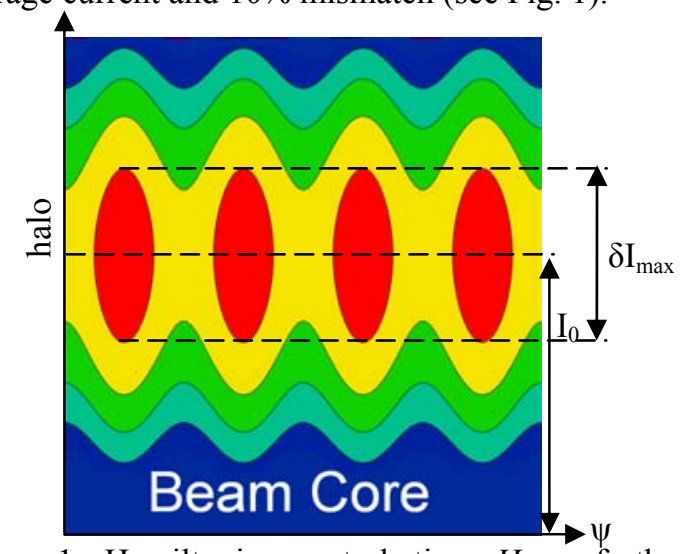

Figure 1: Hamiltonian perturbation $H_{r}$ on forth order resonance conditions $\left(\mathrm{n}=2,4 \theta \approx \mu s, I \approx I_{0}\right)$.

Using the same approach one can obtain Hamiltonian and resonance conditions for resonances caused by beta function oscillation because of the focusing system. These resonances can be clearly expressed in phase space because they are caused by external forces (see Fig. 2).

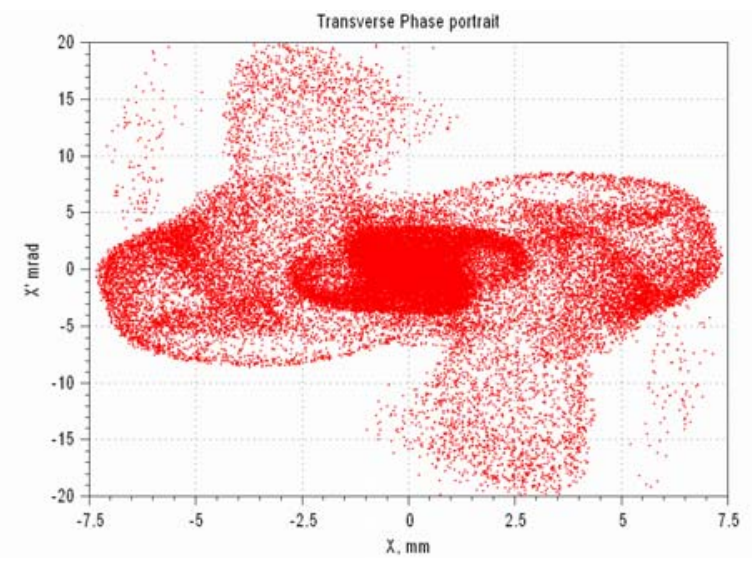

Figure 2: Transverse phase portrait in case of $4^{\text {th }}$ resonance for q-function with particle motion.

\section{CONCLUSIONS}

Using the isolated resonance approach, the theory allowing to predict the halo stabilization due to non-linear features of the space charge resonance was developed. The analytical formula for the resonant deviation of particles in halo, explaining the numerical calculations, were obtained.

\section{REFERENCES}

[1] Yu. Senichev, Mechanical and thermodynamical approach to halo creation problem in high intense beam, HEACC-98, Dubna, 1998, p. 123-125.

[2] N. Bogolyubov and Yu. Mitropol'skij, "Asymptotic Methods in the Theory of non-linear oscillations", Hindustan Publ., Delhi, 1961.

[3] R.Tölle et al., "A superconducting injector linac for COSY" EPAC 02, Paris, 2002, p. 966. 